\title{
The role of aldosterone in kidney diseases and hypertension. Is it worth using mineralocorticoid receptor antagonists in clinical practice?
}

\author{
Rafał Donderski, Jacek Manitius \\ Department of Nephrology, Hypertension and Internal Medicine, Collegium Medicum, Bydgoszcz, Nicolaus Copernicus University, Toruń, Poland
}

\begin{abstract}
Aldosterone is a mineralocorticoid hormone which plays a pivotal role in water and electrolytes balance. Moreover, aldosterone exerts a deleterious influence on the cardiovascular system and kidneys. In this review, we wanted to show mechanisms of aldosterone related organ damage, the role of aldosterone in kidney diseases, hypertension and therapeutic benefits related with aldosterone receptors blockade.

Key words: aldosterone; chronic kidney disease; hypertension; cardiovascular risk, aldosterone antagonists Arterial Hypertens. 2019, vol. 23, no. 1, pages: 1-7 DOI: $10.5603 /$ AH.a2018.0016
\end{abstract}

\section{Introduction}

The renin-angiotensin-aldosterone system (RAAs) plays a key role in the pathogenesis of hypertension, kidney disease and congestive heart failure (CHF). Research on its individual links has been carried out for many years. One of the elements of this system that still raises a lot of controversies is aldosterone. This mineralocorticoid hormone was discovered and described in 1953 by Simpson, Tait and Wetstain. In 1955, dr. Jerome Conn described the case of a woman with severe hypertension, hypokalemia and proteinuria caused by aldosterone-producing adenoma. Many studies from the nineties last century provided proof that aldosterone constituted a factor responsible for myocardial fibrosis and progression of chronic kidney disease (CKD). Subsequent studies in which a well-known al- dosterone antagonist spironolactone was used, showed that this agent added to the classical RAA blockers i.e. losartan and enalapril reduces both proteinuria, blood pressure and decreases left ventricular hypertrophy (LVH) [1, 2]. A number of publications addressed the benefits of blocking the mineralocorticoid receptor in patients with $\mathrm{CHF}$, kidney diseases and resistant hypertension [3]. Mineralocorticoid receptor antagonists have enjoyed a well-established position in pharmacotherapy since the publishing of results of two major clinical trials: the RALES trial (1999) (in which spironolactone was used) and the EPHESUS trial (2003) (in which eplerenone was used) which proved that these mineralocorticoid receptors antagonists had a beneficial effect on decreasing mortality rates in CHF patients $[4,5]$.

Address for correspondence: Rafat Donderski, MD, PhD

Department of Nephrology, Hypertension and Internal Medicine, Collegium Medicum in Bydgoszcz, Nicolaus Copernicus University, Toruń, Poland ul. Skłodowskiej-Curie 9, 85-094 Bydgoszcz, Poland; tel: (+48) 5258540 30, fax: (+48) 525854030 e-mail: rafdon@02.pl 


\section{Aldosterone - synthesis and effects}

Aldosterone is the strongest mineralocorticoid hormone produced both in the zona glomerulosa of the adrenal gland cortex, it is also synthesized extraadrenally in endothelial and vascular smooth muscle cells (VSMC), as well as in the heart and kidneys. Aldosterone demonstrates activity through protein mineralocorticoid receptors type 1 in epithelial cells of the renal tubules, colon, salivary glands, sweat glands, rectal mucosa and urinary bladder. It also affects non-epithelial cells of the central nervous system, as well as cardiomyocytes and VSMC. It serves as an important regulator of the water-electrolyte balance, thus contributing to maintaining the water-electrolyte homeostasis. Acting at the level of distal and collecting tubules, it regulates the $\mathrm{Na}^{+} /$ $\mathrm{K}^{+}$-ATPase-mediated reverse transport of sodium and water and amplifies the inward cellular transport of potassium. In patients with $\mathrm{CKD}$, whose significant clinical problem is the common occurrence of hyperkalemia, aldosterone intensifies the tubular secretion of potassium, enhances the processes of transmineralization, and increases the extrarenal disposal of potassium with stool. Factors affecting the synthesis and regulation of aldosterone secretion are angiotensin II, endothelin, ACTH, hyperkalemia (major regulatory factor in patients with kidney failure) and hypovolemia $[6,7]$.

Classical effects of aldosterone are associated with its influence on the water-electrolyte balance. It causes hypervolemia, hypokalemia, hypomagnesemia, hypochloremia, and metabolic alkalosis. Moreover, aldosterone exerts multidirectional pleiotropic effects falling far beyond the classical scope. It aggravates cardiovascular system fibrosis, fibrosis of renal interstitial tissue, vasoconstriction, endothelial dysfunction, affects and the hemostatic system and enhances inflammation [7]. These effects contribute to the progression of cardiovascular and renal damage. They are particularly severe in the case of high sodium consumption. Effects resulting from the excess of aldosterone are presented in Table I.

\section{Aldosterone and cardiovascular damage in patients with CKD}

Cardiovascular diseases are the main cause of increased mortality rates in CKD patients. This fact is well-known since the 1970s when first reports concerning this matter were published by Linder et al. [8]. Aggravation of atherosclerosis, also common in younger CKD patients results in a 10 to 12 -fold higher risk of acute coronary episodes and death as
Table I. Aldosterone and its multidirectional effects

\begin{tabular}{l}
\hline Aldosterone - classical effects \\
\hline Influence on the water-electrolyte balance (sodium and water \\
retention, hypokalemia, hypochloremia, hypomagnesemia, metabolic \\
alkalosis) \\
Intravascular volume increase and participation in the regulation \\
of arterial blood pressure \\
\hline Aldosterone - pleiotropic effects \\
\hline Synthesis of collagen, TGF- $\beta$ activation, aggravation of heart, \\
vessels and kidney interstitial fibrosis \\
Influence on the hemostatic system, PAl-1 activation \\
Endothelial dysfunction. \\
Proinflammatory action and immunomodulation action \\
Effect on bone metabolism \\
Aggravation of oxidative stress \\
Effect on the concentration of lipids \\
Kidney damage - mesangial cells proliferation and podocytes \\
damage \\
TGF- $\beta$ - transforming growth factor $\beta$; PAl-1 - plasminogen activator inhibitor-1
\end{tabular}

compared to the general population. The more frequent occurrence of cardiovascular diseases (CVD) is a result of the influence of both traditional and non-traditional risk factors i.e. peculiar for kidney disease, including anaemia, disturbances in calciumphosphate management (CKD-MBD), vessels calcification, the presence of an arteriovenous fistula, hyperhomocysteinemia and others [9].

Aldosterone excess and its local synthesis is a potent stimulator of myocardial fibrosis in CKD patients with coexisting CHF. Increased local synthesis of collagen type I (PINP) and III (PIIINP) was observed in this population. This applies particularly to patients after myocardial infarction (MI) who present post-infarction heart failure, in whom aldosterone plays a crucial role in the post-infarction left ventricle (LV) remodelling [10]. Aldosterone related cardiac fibrosis causes its indirect proarrhythmic effects. Aldosterone also contributes to the impairment of insulin secretion. Increased levels of glucose in diabetic patients aggravate the aldosterone-induced processes of cardiomyocytes hypertrophy [11].

Moreover, excess aldosterone affects $\mathrm{LVH}$ in patients with hypertension and CKD both on conservative and renal replacement therapy treatment (RRT) [12]. It also contributes to the aggravation of endothelial damage, inhibition of nitric oxide synthase (iNOS) activity, and impairment of endothelium-dependent vasodilation [13]. Apart from being involved in cardiac remodelling, aldosterone also plays a role in the process of vascular remodelling, leading to increased arterial stiffness related to the accumulation of fibronectin [14].

The deleterious effects of aldosterone excess are also associated with sodium retention, hypokalemia, 
influence on blood pressure, impairment of the function of baroreceptors, and increased adrenergic activity in patients with CHF [15]. Heart and vascular damage induced by aldosterone excess cause the local inflammatory response, the activation of various cytokines and inflammatory mediators such as MCP-1, COX-2, osteopontin [16]. Moreover, aldosterone aggravates oxidative stress and impaired tissue insulin sensitivity of the vascular wall. It contributes to the acceleration of atherosclerosis. It is also worth to mention about the role of aldosterone in the metabolism of bone tissue and its influence on the immune system. According to the many of aldosterone action, aldosterone antagonists usage is a part of cardio- and angioprotection in CKD patients.

\section{Role of aldosterone in the progression of chronic kidney disease (CKD)}

Inhibiting the progression of kidney diseases associated with the use of aldosterone receptor antagonists is a consequence of the effects of those drugs on numerous components of pathophysiological processes responsible for progressive and irreversible renal damage. The only undeniably significant element inhibiting the process of renal damage is the reduction of blood pressure. Hypertension is a modifiable factor undoubtedly leading to increased urinary protein excretion and proteinuria-related glomerular filtration impairment. It must also be emphasized that the nephroprotective effect of those drugs, that is inhibiting the progression of kidney disease, seems to be independent of the reduction of blood pressure, which has been proved in a number of clinical trials $[17,18]$. It has recently been stressed that this beneficial effect might be a consequence of the nongenomic action of these drugs, which is independent of the direct action of aldosterone antagonists on its nuclear receptor. There is extensive data indicating that the nongenomic action of this drug group takes place through the membrane receptor [19]. The nephroprotective effects of aldosterone receptor antagonists, also resulting from their influence on the membrane receptor, might be strongly associated with their beneficial effects on endothelial function [20].

The role of aldosterone in the process of kidney damage should be investigated in the context of systemic effects, as well as local effects within the organ itself. In a study of biopsy specimens obtained from patients with primary and secondary glomerulopathies, it was found that mRNA expression for aldosterone receptors increased proportionally to the levels of urinary protein excretion. Moreover, in patients with the highest albumin excretion rates, increased mRNA expression was also found within the interstitium, while in patients whose protein excretion rates were lower, mRNA expression was mainly associated with the area of distal and collecting tubules. In the context, it should be noted that along with the increase in mRNA expression for the aldosterone receptor in individual fragments of biopsy specimens, a proportional increase in $\mathrm{mRNA}$ expression was revealed for MCP-1 and TGF $\beta-1$, which are key factors influencing the development of renal tubulointerstitial fibrosis. The increase in mRNA expression for the aldosterone receptor was a local response to nephron exposure to the level of proteinuria and was not determined by the serum concentration of aldosterone [21]. Another mechanism responsible for the occurrence of proteinuria is podocyte injury associated with the overexpression of aldosterone receptors within the glomerulus. However, increased aldosterone receptor expression in podocytes is independent of the serum concentration of aldosterone $[22,23]$. Notwithstanding the abovementioned data, there is a lot of evidence indicating that the efficiency of aldosterone receptor antagonists administration in proteinuric patients is also affected by the degree of aldosteronemia. This can be inferred from data revealing that a decrease in protein excretion rates resulting from the use of spironolactone remains in simple correlation with the serum concentration of aldosterone [24].

The interest in possible applications of aldosterone antagonists in the field of nephroprotection inspires researchers to undertake further studies, including a large proportion of clinical trials. Regardless of the acknowledged risk associated with this therapy (such as hyperkalemia), drugs from this group have been more and more commonly used in the treatment of CKD [25].

\section{Therapeutic possibilities associated with mineralocorticoid receptor blockade in CKD and hypertension patients}

The use of angiotensin-converting enzyme inhibitors (ACE-I) and angiotensin II receptor blockers (ARB) in clinical practice has become a commonly accepted form of treatment of cardiovascular diseases (CVD) and kidney diseases accompanied by proteinuria or renal function impairment. In numerous studies published also by Polish authors, there had been shown that these drugs inhibit the progression 
of CKD into the end stage phase and reduce proteinuria [26]. A decrease in the synthesis of aldosterone is often observed in the first months of their administration, however, due to the so-called aldosterone escape phenomenon, their prolonged administration does not lead to a further decrease in aldosterone synthesis. It seems that this problem may be observed in approximately $30-50 \%$ of patients treated with ACE-I/ARB [27, 28]. Complete control over the RAA system requires the supplementary drugs from the group of mineralocorticoid receptor antagonists (MRAs). In clinical practice, the most commonly used ones are spironolactone and eplerenone. The latter is more selective for the mineralocorticoid receptor and demonstrates fewer adverse effects related to its influence on hormonal balance (lower affinity for androgen and progesterone receptors).

Recently, a new mineralocorticoid receptor antagonist (MRA) - finerenone has been introduced in treatment. It is a nonsteroidal drug which does not induce hyperkalemia [29]. The above mentioned MRA has been successfully used in CKD patients both on conservative treatment and on RRT. A metaanalysis of 18 randomized clinical trials in 1786 diabetic nephropathy patients in whose standard therapy with ACE-Is or ARBs use was supplemented with the administration of MRA, demonstrated a beneficial effect of this combination and decrease in the urinary albumin excretion rate, albumin/creatinine ratio (ACR), and blood pressure. There was no improvement in the glomerular filtration rate (GFR). An increased risk of hyperkalemia was observed [30]. Edwards et al. in CRIB-II study found that 10 weeks spironolactone treatment added to ACE-I or ARB caused reduction of the left ventricular mass index (LVMI) in non-diabetic CKD stage 2 and 3 patients [31]. Currently conducted BARACK-D trial (Benefits of Aldosterone Receptor Antagonism in Chronic Kidney Disease) in 2616 patients with stage $3 \mathrm{~b}$ CKD will compare the efficacy of spironolactone $25 \mathrm{mg}$ once daily in addition to routine care (ACE-I/ARB) on mortality and cardiovascular outcomes versus routine care alone [32]. Combined treatment with ACE-Is/ARBs and aldosterone antagonist is possible in CKD patients, but it requires attention and a frequent potassium level control. Practically speaking, it would be inadvisable to start treatment with MRA in CKD patients and eGFR $<30 \mathrm{~mL} / \mathrm{min}$ or serum potassium level $>5.0 \mathrm{mmol} / \mathrm{L}$. The risk of hyperkalemia related to the treatment significantly limits wider aldosterone antagonists use in CKD patients. It is worth to mention that a new pharmacological agent is now available to reduce hyperkalemia in CKD. Patiromer is a novel oral potassium binder indicated for hyperkalemia treatment in CKD patients on conservative treatment. The initial dose is $8.4 \mathrm{~g}$ p.o. daily and it may be adjusted based on the potassium level up to $25.2 \mathrm{~g}$ daily. Patiromer added on standard treatment may facilitate aldosterone receptor antagonist introduction to therapy with other RAA inhibitors [33]. Advantages of the administration of aldosterone antagonists in the improvement of heart and vascular function have also been described in dialysis patients [34]. Nitta et al. in a group of hemodialyzed patients, demonstrated positive effects of spironolactone on the inhibition of vascular calcification. He indicated that this effect was associated with a decrease of osteopontin expression in the vascular wall [35]. In our own study, conducted in a group of 22 peritoneal dialysis patients, an increased plasma concentration of osteopontin after 12 months of spironolactone treatment in a dose of 50 mg daily was demonstrated. This effect can be considered beneficial because osteopontin besides its other actions is also considered to be a potent inhibitor of vessels calcification. There was no hyperkaliemia during spironolactone administration [36]. Vukusich et al. in non-diabetic, hemodialyzed CKD patients demonstrated a beneficial effect of 2-year spironolactone administration 3 times a week at a daily dose of $50 \mathrm{mg}$ for stabilization and even a reduction of the IMT complex thickness of carotid arteries [37]. The currently conducted STOP-CKD study in the population of CKD stage 3 patients in general practitioners (GP) care will show the influence of low doses of spironolactone on the reduction of arterial stiffness [38]. As it was mentioned above aldosterone antagonists administration is related to hyperkalemia risk. Chua et al performed a meta-analysis of 6 randomized clinical trials in 154 hemodialyzed patients. They focused on the safety of spironolactone use in an aspect of hyperkalemia. The authors concluded that spironolactone seems to be safe in a short-term therapy. Strict potassium control is mandatory [39]. In DOHAS study 309 hemodialyzed patients were randomized to either placebo group or spironolactone group and treated with $25 \mathrm{mg}$ of spironolactone daily for3 years. In patients treated with spironolactone, there was a statistically significant reduction of risk of death or cardiovascular hospitalization or death of general reasons in comparison to the placebo group. The incidence of life-threateninghyperkalemia was (potassium level $>6.5 \mathrm{mmol} / \mathrm{L}$ ) $1.9 \%$ and gynecomastia occurred in $10 \%$ of the spironolactone patients [40]. The future perspective of spironolactone treatment in hemodialyzed patients will be available soon as a result of another new study - ALCHEMIST study (Aldosterone Antagonist Chronic 
Hemodialysis Interventional Survival Trial, ALCHEMIST) [41]. Moreover, in many studies benefits of aldosterone antagonists in peritoneal dialysis patients were confirmed. These both are a description of individual case reports and randomized trials involving larger groups of patients. Hausmann et al. described an improvement in cardiac function manifested by an increase of ejection fraction from $32 \%$ to $46 \%$ in a patient receiving peritoneal dialysis treatment and spironolactone at a dose of $25 \mathrm{mg} /$ day administered for 10 months [42]. Ito et al. in 2014 published the results of his study of 158 peritoneal dialysis patients. The combined treatment with ACE-I or ARB and spironolactone at a dose of $25 \mathrm{mg}$ per day caused a significant improvement in left ventricular ejection fraction after 2 years of treatment compared to the control group. Left ventricular mass reduction (LVMI) was found after 6 months of therapy. There were no significant changes in risk of death [43]. The excess of aldosterone in peritoneal dialysis patients aggravates cardiovascular damage and contributes to the progressive failure of the peritoneal membrane. This may be related with accelerated fibrosis of the peritoneal membrane. Kolesnyk et al. showed a beneficial effect of ARB treatment in halting the progression of the peritoneal membrane sclerosis and calcification [44]. Vazquez-Rangel et al. in a group of 9 patients on peritoneal dialysis treated with spironolactone at a dose of $25 \mathrm{mg}$ observed in histopathological evaluation of peritoneal membrane specimens reduction in severity of inflammation and collagen accumulation [45]. It is worth to mention that aldosterone antagonists treatment in patients receiving peritoneal dialysis seems to be safe, because dialysis fluid does not contain potassium and the risk of lifethreatening hyperkalemia is significantly diminished. Aldosterone antagonists have a well-established position in the treatment of secondary hypertension associated with primary hyperaldosteronism (preparation for a surgical procedure in adrenocortical adenoma or unilateral adrenocortical hyperplasia, longterm treatment in patients with primary hyperaldosteronism resulting from bilateral adrenal hyperplasia) [46]. It should also be mentioned that aldosterone antagonists have been demonstrated to have beneficial effects in the treatment of resistant hypertension i.e. a clinical situation in which, despite lifestyle modifications and treatment with 3 antihypertensive drugs in optimal doses, including a diuretic, optimal blood pressure control is unattainable. Many previously published studies had proved the usefulness of aldosterone antagonists in patients with resistant hypertension and the effectiveness of this group of drugs in the reduction of both systolic and dia- stolic blood pressure. Advantages of aldosterone antagonists use in the treatment of resistant hypertension had been shown in individuals with visceral obesity or obstructive sleep apnea syndrome (OSAS). It was also described that a large proportion of cases (approximately 23-25\%) of primary hyperaldosteronism are also found in patients with refractory hypertension $[47,48]$. In obese patients with hypertension, an elevated serum aldosterone level was observed in comparison to patients with normal body weight and without hypertension. It may result from increased production of linoleic acid (EKODE) in adipocytes of obese patients. Linoleic acid has been shown to stimulate the secretion of aldosterone in the adrenal cortex [49]. There also have been demonstrated interactions between parathyroid hormone (PTH) and aldosterone. PTH increases aldosterone secretion by affecting the RAA system. Both hormones have a markedly adverse effect on the cardiovascular system [50]. The strong position of spironolactone was recently confirmed in the completed randomized trial - Pathway-2. This study has shown that spironolactone was the most effective drug, more effective than doxazosin or bisoprolol as an add-on drug for treatment of really resistant hypertension [51].

In conclusion, aldosterone excess through its metabolic effects, intensification of vasoconstriction, inflammation or fibrosis is undoubtedly a factor responsible for cardiovascular system damage and progression of CKD. Hence, it is of utmost importance to reduce its effects and to use aldosterone antagonists extensively in selected groups of patients. Bolignano et al. in the meta-analysis (published in 2014) of studies with aldosterone antagonists added on ACE-I/ARB treatment found that these agents may cause a decrease of proteinuria and blood pressure but their influence on cardiovascular mortality is rather questionable. Despite that statement currently conducted interventional studies will show final benefits of these agents in kidney disease patients [52].

Nothing to declare.

\section{Conflict of interest}

\section{References}

1. Weber KT, Villarreal D. Aldosterone and antialdosterone therapy in congestive heart failure. Am J Cardiol. 1993; 71(3): 3A-11A, indexed in Pubmed: 8422002.

2. Hollenberg NK. Aldosterone in the development and progression of renal injury. Kidney Int. 2004; 66(1): 1-9, doi: 10.1111/j.15231755.2004.00701.x, indexed in Pubmed: 15200407.

3. Epstein M. Aldosterone as a determinant of cardiovascular and renal dysfunction. J R Soc Med. 2001; 94(8): 378-383, doi: 10.1177/01 4107680109400803 , indexed in Pubmed: 11461980. 
4. Pitt B, Zannad F, Remme WJ, et al. The effect of spironolactone on morbidity and mortality in patients with severe heart failure. Randomized Aldactone Evaluation Study Investigators. N Engl J Med. 1999; 341(10): 709-717, doi: 10.1056/NEJM199909023411001, indexed in Pubmed: 10471456.

5. Ferreira JP, Duarte K, McMurray JJV, et al. Eplerenone Post-Acute Myocardial Infarction Heart Failure Efficacy and Survival Study Investigators. Eplerenone, a selective aldosterone blocker, in patients with left ventricular dysfunction after myocardial infarction. N Engl J Med. 2003; 348(14): 1309-1321, doi: 10.1056/NEJMoa030207, indexed in Pubmed: 12668699.

6. Musso CG. Potassium metabolism in patients with chronic kidney disease (CKD), Part I: patients not on dialysis (stages 3-4). Int Urol Nephrol. 2004; 36(3): 465-468, doi: 10.1007/s11255-004-6193-z, indexed in Pubmed: 15783125.

7. Funder JW. Aldosterone and Mineralocorticoid Receptors Physiology and Pathophysiology. Int J Mol Sci. 2017; 18(5): 1032, doi: 10.3390/ijms18051032, indexed in Pubmed: 28492512.

8. Lindner A, Charra B, Sherrard DJ, et al. Accelerated atherosclerosis in prolonged maintenance hemodialysis. N Engl J Med. 1974; 290(13): 697-701, doi: 10.1056/NEJM197403282901301, indexed in Pubmed: 4813742.

9. Lai S, Dimko M, Galani A, et al. Early markers of cardiovascular risk in chronic kidney disease. Ren Fail. 2014; 37(2): 254-261, do i: $10.3109 / 0886022 x .2014 .982489$.

10. Funder J. Mineralocorticoids and cardiac fibrosis: the decade in review. Clin Exp Pharmacol Physiol. 2001; 28(12): 10021006, doi: $10.1046 /$ j.1440-1681.2001.03586.x, indexed in Pubmed: 11903303.

11. Sato A, Funder JW. High glucose stimulates aldosteron-induced hyperthrophy via type I mineralocorticoid receptors in neonatal rat cardiomiocytes. Endocrinology. 1996; 137(10): 4145-4153.

12. Feniman De Stefano GM, Zanati-Basan SG, De Stefano LM, et al. Aldosterone is associated with left ventricular hypertrophy in hemodialysis patients. Ther Adv Cardiovasc Dis. 2016; 10(5): 304-313, doi: 10.1177/1753944716644583, indexed in Pubmed: 27122492.

13. Abiose AK, Mansoor GA, Barry M, et al. Effect of spironolactone on endothelial function in patients with congestive heart failure on conventional medical therapy. Am J Cardiol. 2004; 93(12): 1564-1566, doi: 10.1016/j.amjcard.2004.03.015, indexed in Pubmed: 15194040.

14. Leibovitz E, Ebrahimian T, Paradis P, et al. Aldosterone induces arterial stiffness in absence of oxidative stress and endothelial dysfunction. J Hypertens. 2009; 27(11): 2192-2200, doi: 10.1097/ HJH.0b013e328330a963, indexed in Pubmed: 19654560.

15. Miller AB. Aldosterone antagonism in heart failure. Vasc Health Risk Manag. 2007; 3(5): 605-609, indexed in Pubmed: 18078011.

16. Gilbert KC, Brown NJ. Aldosterone and inflammation. Curr Opin Endocrinol Diabetes Obes. 2010; 17(3): 199-204, indexed in Pubmed: 20422780.

17. Currie G, Taylor AHM, Fujita T, et al. Effect of mineralocorticoid receptor antagonists on proteinuria and progression of chronic kidney disease: a systematic review and meta-analysis. BMC Nephrol. 2016; 17(1): 127, doi: 10.1186/s12882-016-0337-0, indexed in Pubmed: 27609359.

18. Kato S, Maruyama S, Makino $\mathrm{H}$, et al. Anti-albuminuric effects of spironolactone in patients with type 2 diabetic nephropathy: a multicenter, randomized clinical trial. Clin Exp Nephrol. 2015; 19(6): 1098-1106, doi: 10.1007/s10157-015-1106-2, indexed in Pubmed: 25795029.

19. Wildling L, Hinterdorfer P, Kusche-Vihrog K, et al. Aldosterone receptor sites on plasma membrane of human vascular endothelium detected by a mechanical nanosensor. Pflugers Arch. 2008; 458(2): 223-230, doi: 10.1007/s00424-008-0615-1.

20. Toto RD. Aldosterone blockade in chronic kidney disease: can it improve outcome? Curr Opin Nephrol Hypertens. 2010; 19(5): 444-449, doi: 10.1097/MNH.0b013e32833ce6d5, indexed in Pubmed: 20625290.

21. Quinkler M, Zehnder D, Eardley KS, et al. Increased expression of mineralocorticoid effector mechanisms in kidney biopsies of patients with heavy proteinuria. Circulation. 2005; 112(10): 1435-1443, doi: 10.1161/CIRCULATIONAHA.105.539122, indexed in Pubmed: 16145013

22. Shibata S, Fujita T. Mineralocorticoid receptors in the pathophysiology of chronic kidney diseases and the metabolic syndrome. Mol Cell Endocrinol. 2012; 350(2): 273-280, doi: 10.1016/j. mce.2011.07.018, indexed in Pubmed: 21820485.

23. Shibata S, Ishizawa K, Uchida S. Mineralocorticoid receptor as a therapeutic target in chronic kidney disease and hypertension. Hypertens Res. 2017; 40(3): 221-225, doi: 10.1038/hr.2016.137, indexed in Pubmed: 27760997.

24. Bianchi S, Bigazzi R, Campese VM. Long-term effects of spironolactone on proteinuria and kidney function in patients with chronic kidney disease. Kidney Int. 2006; 70(12): 2116-2123, doi: 10.1038/ sj.ki.5001854, indexed in Pubmed: 17035949.

25. Ma TW, Szeto CC. Mineralocorticoid Receptor Antagonist for Renal Protection. Ren Fail. 2012; 34(6): 810-817, doi: 10.3109/088602 2x.2012.672156, indexed in Pubmed: 22463731.

26. Tylicki L, Lizakowski S, Rutkowski P, et al. The enhanced reninangiotensin-aldosteron system pharmacological blockade - which is the best?. Kidney Blood Press Res. 2012; 36(1): 335-343, doi: 10.1159/000343391, indexed in Pubmed: 23235363.

27. Struthers $\mathrm{AD}$. The clinical implications of aldosterone escape in congestive heart failure. Eur J Heart Fail. 2004; 6(5): 539-545.

28. Sato A, Sato A, Saruta T, et al. Effectiveness of aldosterone blockade in patients with diabetic nephropathy. Hypertension. 2003; 41(1): 64-68, doi: 10.1161/01.hyp.0000044937.95080.e9, indexed in Pubmed: 12511531.

29. Dutzmann J, Musmann RJ, Haertlé M, et al. The novel mineralocorticoid receptor antagonist finerenone attenuates neointima formation after vascular injury. PLoS One. 2017; 12(9): e0184888, doi: 10.1371/journal.pone.0184888, indexed in Pubmed: 28926607.

30. Sun LJ, Sun YN, Shan JP, et al. Effects of mineralocorticoid receptor antagonists on the progression of diabetic nephropathy. J Diabetes Investig. 2017; 8(4): 609-618, doi: 10.1111/jdi.12629, indexed in Pubmed: 28107779.

31. Edwards NC, Steeds RP, Stewart PM, et al. Effect of spironolactone on left ventricular mass and aortic stiffness in early-stage chronic kidney disease: a randomized controlled trial. J Am Coll Cardiol. 2009; 54(6): 505-512, doi: 10.1016/j.jacc.2009.03.066, indexed in Pubmed: 19643310.

32. Hill N, Lasserson D, Thompson B, et al. Benefits of Aldosterone Receptor Antagonism in Chronic Kidney Disease (BARACK D) trial-a multi-centre, prospective, randomised, open, blinded end-point, 36-month study of 2,616 patients within primary care with stage $3 \mathrm{~b}$ chronic kidney disease to compare the efficacy of spironolactone $25 \mathrm{mg}$ once daily in addition to routine care on mortality and cardiovascular outcomes versus routine care alone: study protocol for a randomized controlled trial. Trials. 2014; 15: 160, doi: 10.1186/1745-6215-15-160.

33. Weir MR, Bakris GL, Bushinsky DA, et al. OPAL-HK Investigators. Patiromer in patients with kidney disease and hyperkalemia receiving RAAS inhibitors. N Engl J Med. 2015; 372(3): 211-221, doi: 10.1056/NEJMoa1410853, indexed in Pubmed: 25415805.

34. Covic A, Gusbeth-Tatomir P, Goldsmith DJA. Is it time for spironolactone therapy in dialysis patients? Nephrol Dial Transplant. 2006; 21(4): 854-858, doi: 10.1093/ndt/gfk086, indexed in Pubmed: 16449287.

35. Nitta K, Akiba T, Nihei H. Aldosterone blockade and vascular calcification in hemodialysis patients. Am J Med. 2003; 115(3): 250a, doi: 10.1016/s0002-9343(03)00293-6.

36. Donderski R, Stróżecki P, Sulikowska B, et al. Aldosterone antagonist therapy and its relationship with inflammation, fibrosis, thrombosis, mineral-bone disorder and cardiovascular complications in peritoneal dialysis (PD) patients. Int Urol Nephrol. 2017; 49(10): 1867-1873, doi: 10.1007/s1 1255-017-1655-2, indexed in Pubmed: 28710615.

37. Vukusich A, Kunstmann S, Varela C, et al. A randomized, doubleblind, placebo-controlled trial of spironolactone on carotid intima media thickness in nondiabetic hemodialysis patients. Clin J Am Soc 
Nephrol. 2010; 5(8): 1380-1387, doi: 10.2215/CJN.09421209, indexed in Pubmed: 20522535.

38. Khai PNg, Jain P, Heer G, et al. Spironolactone to prevent cardiovascular events in early-stage chronic kidney disease (STOP-CKD): study protocol for a randomized controlled pilot trial. Trials. 2014; 15: 158, doi: $10.1186 / 1745-6215-15-158$, indexed in Pubmed: 24886272.

39. Chua D, Lo A, Lo C. Spironolactone use in heart failure patients with end-stage renal disease on hemodialysis: is it safe? Clin Cardiol. 2010; 33(10): 604-608, doi: 10.1002/clc.20838, indexed in Pubmed: 20960534.

40. Matsumoto Y, Mori Y, Kageyama S, et al. Spironolactone reduces cardiovascular and cerebrovascular morbidity and mortality in hemodialysis patients. J Am Coll Cardiol. 2014; 63(6): 528-536, doi: 10.1016/j.jacc.2013.09.056, indexed in Pubmed: 24184249.

41. Bomback AS. Mineralocorticoid Receptor Antagonists in End-Stage Renal Disease: Efficacy and Safety. Blood Purif. 2016; 41(1-3): 166170, doi: 10.1159/000441262, indexed in Pubmed: 26765793.

42. Fenwick S, Bell GM. Aldactone therapy in a peritoneal dialysis patient. Nephrol Dial Transplant. 2003; 18(6): 1232-1232, doi: $10.1093 / \mathrm{ndt} / \mathrm{gfg} 098$.

43. Hausmann MJ, Liel-Cohen N. Aldactone therapy in a peritoneal dialysis patient with decreased left ventricular function. NDT. 2002; 17(11): 2035-2036, doi: https://doi.org/10.1093/ndt/17.11.2035.

44. Ito Y, Mizuno M, Suzuki Y, et al. Nagoya Spiro Study Group. Long-term effects of spironolactone in peritoneal dialysis patients. J Am Soc Nephrol. 2014; 25(5): 1094-1102, doi: 10.1681/ ASN.2013030273, indexed in Pubmed: 24335969.

45. Kolesnyk I, Noordzij M, Dekker FW, et al. A positive effect of AII inhibitors on peritoneal membrane function in long-term PD patients. Nephrol Dial Transplant. 2009; 24(1): 272-277, doi: 10.1093/ndt/ gfn421, indexed in Pubmed: 18676349.

46. Vazquez-Rangel A, Soto V, Escalona M, et al. Spironolactone to prevent peritoneal fibrosis in peritoneal dialysis patients: a randomized controlled trial. Am J Kidney Dis. 2014; 63(6): 1072-1074, doi: 10.1053/j.ajkd.2014.01.426, indexed in Pubmed: 24613053.

47. Siewaszewicz E. Antagoniści aldosteronu w leczeniu nadciśnienia tętniczego. AHT. 2010; 14(3): 216-226.

48. Yugar-Toledo JC, Modolo R, Moreno H, et al. Managing resistant hypertension: focus on mineralocorticoid-receptor antagonists. Vasc Health Risk Manag. 2017; 13: 403-411, doi: 10.2147/VHRM. S138599.

49. Dahal K, Kunwar S, Rijal J, et al. The Effects of Aldosterone Antagonists in Patients With Resistant Hypertension: A Meta-Analysis of Randomized and Nonrandomized Studies. Am J Hypertens. 2015; 28(11): 1376-1385, doi: 10.1093/ajh/hpv031, indexed in Pubmed: 25801902 .

50. Goodfriend TL, Ball DL, Egan BM, et al. Epoxy-keto derivative of linoleic acid stimulates aldosterone secretion. Hypertension. 2004; 43(2): 358-363, doi: 10.1161/01.HYP.0000113294.06704.64, indexed in Pubmed: 14718355.

51. Tomaschitz A, Ritz E, Pieske B, et al. Aldosterone and parathyroid hormone: a precarious couple for cardiovascular disease. Cardiovasc Res. 2012; 94(1): 10-19, doi: $10.1093 / \mathrm{cvr} / \mathrm{cvs} 092$, indexed in Pubmed: 22334595.

52. Williams B, MacDonald TM, Morant S, et al. British Hypertension Society's PATHWAY Studies Group. Spironolactone versus placebo, bisoprolol, and doxazosin to determine the optimal treatment for drug-resistant hypertension (PATHWAY-2): a randomised, double-blind, crossover trial. Lancet. 2015; 386(10008): 2059-2068, doi: 10.1016/S0140-6736(15)00257-3, indexed in Pubmed: 26414968.

53. Bolignano D, Palmer SC, Navaneethan SD, et al. Aldosterone antagonists for preventing the progression of chronic kidney disease. Cochrane Database Syst Rev. 2014(4): CD007004, doi: 10.1002/14651858.CD007004.pub3, indexed in Pubmed: 24782282. 